\title{
Kinetic Characterization of Tyrosinase-catalyzed Oxidation of Four Polyphenols*
}

\author{
Wan-yu LIU ${ }^{1 \dagger}$, Cong-ming ZOU ${ }^{2 \dagger}$, Jian-hua $\mathrm{HU}^{1}$, Zi-jun XU ${ }^{1}$, Lu-qin $\mathrm{SI}^{1}$, Jun-jun LIU $^{1 \#}$, Jian-geng HUANG ${ }^{1 \#}$

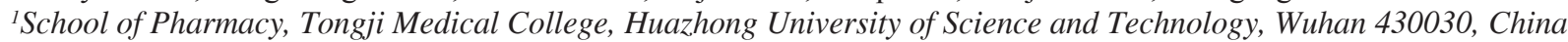 \\ ${ }^{2}$ Yunnan Academy of Tobacco Agricultural Sciences, Kunming 650021, China
}

(C) The Author(s) 2020, corrected publication July 2020

\begin{abstract}
Summary: Phenolic compounds such as chlorogenic acid, cryptochlorogenic acid, neochlorogenic acid and caffeic acid are widely distributed in fruits, vegetables and traditional Chinese medicines with a wide range of biological activities. Tyrosinase plays a critical role in the food industry, but recent studies have proposed unexplored aspects of clinical application. Tyrosinase-catalyzed oxidation of four polyphenols as well as its underlying mechanism remains unclear. In the current work, we investigated the kinetic properties of tyrosinase-catalyzed oxidation of the four polyphenols of interest. To measure the unstable o-quinone products, an analytical method using 3-methyl-2-benzothiazolinone hydrazone (MBTH) was established. The optimal incubation time, buffer $\mathrm{pH}$, temperature and enzyme concentration for the enzyme activity in the presence of each polyphenol of interest were investigated. Under the final optimized conditions, the kinetics and substrate specificity of four polyphenols were examined. Kinetic data showed that tyrosinase had the greatest substrate affinity to chlorogenic acid compared with its isomers and caffeic acid. The catalytic efficiency with chlorogenic acid was 8 - to 15 -fold higher than that with the other 3 polyphenols. Molecular docking study demonstrated that the tight binding of chlorogenic acid at the peripheral site should be the major reason for the specificity to chlorogenic acid. In light of this, the rational design of high-affinity inhibitors against tyrosinase may focus on the binding of both the $\mathrm{Cu}$ site and peripheral site. This study will supply a basis for the selection of phenolic acids in food industry and health care.
\end{abstract}

Key words: polyphenols; tyrosinase; kinetic characterization; molecular docking

Daily diet plays an important role in disease prevention. Evidence shows that people can benefit from plant phenolic compounds which are often absorbed from the $\operatorname{diet}^{[1]}$. Phenolic compounds are ubiquitous in plants and consist of a large number of secondary metabolites derived from pentose phosphate, shikimate and phenylpropanoid pathways ${ }^{[2]}$. The composition and amount of phenolic substances are

The original version of this article was revised due to a retrospective Open Access order.

Wan-yu LIU, E-mail: m201875230@hust.edu.cn; Congming ZOU, E-mail: zoucongmingzcm@163.com

${ }^{\dagger}$ The authors contributed equally to this study.

${ }^{\#}$ Corresponding authors, Jun-jun LIU, E-mail: junjun.liu@ hust.edu.cn; Jian-geng HUANG, E-mail: jiangenghuang@, hust.edu.cn

"The study was supported by grants from the National Natural Science Foundation of China (No. 81773811), Yunnan Applied Basic Research Project (No. 2017FB074), the Yunnan Provincial Tobacco Monopoly Bureau Grants (No. 2017YN09) and the Fundamental Research Funds for the Central Universities (No. 2020kfyXGYJ061). determined by a variety of genetic and environmental factors $^{[3]}$. Phenolics not only contribute to the color and sensory characteristics of vegetables and fruits, but also provide protection against pathogens and predators. More importantly, phenolic chemicals are a major source of the intake of natural antioxidants in the human $\operatorname{diet}^{[4]}$.

Typical plant-derived phenolic compounds occurring in the human diet include flavonoids, phenolic acids, tannins, stilbens and lignans, etc. It is worthy to note that major classes of phenolic acids are hydroxycinnamic acids and hydroxybenzoic acids. The main polyphenolic representatives of hydroxycinnamic acids are chlorogenic acid and caffeic acid ${ }^{[5]}$. The latter is found in foods largely as an ester form with quinic acid called chlorogenic acid ${ }^{[6]}$. Chlorogenic acid, also known as 3-O-caffeoyl quinine, is widely distributed in fruits (apple), vegetables (potato), drinks such as tea, coffe $e^{[7]}$, wine ${ }^{[8]}$, traditional Chinese medicines such as honeysuckle ${ }^{[9]}$, gardenia ${ }^{[10]}$ and even tobacco ${ }^{[11]}$. According to different esterification sites of quinine, 4-O-caffeoyl quinine (cryptochlorogenic 
acid) and 5-O-caffeoyl quinine (neochlorogenic acid) are two important isomers of chlorogenic acid. Recently, an increasing number of research papers have been published to ascertain the nutritional benefits and physiological effect ${ }^{[8]}$. Scientific evidence shows that chlorogenic acid has a wide range of biological activities, such as antioxidant ${ }^{[12]}$ and anti-mutagenesis ${ }^{[13]}$, cardiovascular protection ${ }^{[14]}$, antibacteria and antivirus ${ }^{[15]}$, immune regulation, lowering blood glucose and blood lipids ${ }^{[16]}$. Moreover, pharmacological studies have also demonstrated that both cryptochlorogenic acid and neochlorogenic acid exhibit excellent antioxidative, antibacterial, antiviral and antipyretic activities ${ }^{[17]}$. In addition, caffeic acid has also demonstrated antioxidant activity in the prevention of premature aging and antimicrobial activity in the treatment of dermal diseases ${ }^{[18]}$.

Tyrosinase is a copper-containing monooxygenase, widely distributed in plants, micro-organisms and mammals ${ }^{[19]}$. Tyrosinase catalyzes two types of reaction, by which monophenols and o-diphenols are oxidized to o-diphenols and o-quinones, respectively ${ }^{[20]}$. The role of tyrosinase for triggering enzymatic browning reactions has been well established in the food industry. Searching potent tyrosinase inhibitors is of great importance for postponing the discoloration process. To gain this goal, a large number of chemicals from both natural and synthetic sources have been tried ${ }^{[21-24]}$. However, more efforts are still required to identify better inhibitors without obvious adverse side effect. Recently, a variety of studies have proposed several previously unexplored aspects of tyrosinase in clinical studies. Tyrosinase also plays a key role in the pigments synthesis such as melanin, which regulates the color of hair and $\operatorname{skin}^{[25]}$. Tyrosinase is considered as a target in the treatment of specific dermatological diseases associated with melanin hyperpigmentation ${ }^{[26,27]}$. Tyrosinase is also known as an autoantigen in various autoimmune disease and serves as a marker for vitiligo ${ }^{[28]}$. Despite contradictory opinion concerning the role of tyrosinase in mutagenicity and tumor suppression, melanocytedirected tyrosinase prodrug therapy might offer a highly selective drug delivery approach for malignant melanoma $^{[29,30]}$.

Chlorogenic acid has been reported as a substrate of polyphenol oxidase derived from apple ${ }^{[7]}$ and dill

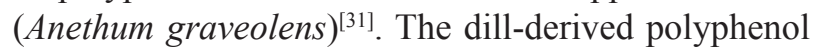
oxidase exhibited better affinity to chlorogenic acid than catechol and dopamine ${ }^{[31]}$. However, very little attention has been devoted to polyphenol oxidase mediated oxidation of the isomers of chlorogenic acid such as cryptochlorogenic acid and neochlorogenic acid. In the current work, we investigated the kinetic properties and the underlying mechanism of tyrosinasecatalyzed oxidation of the four polyphenols of interest, which can provide detailed enzymatic characteristics in food industry and health care.

\section{MATERIALS AND METHODS}

\subsection{Materials}

Mushroom tyrosinase (2687 units/mg) was purchased from Sigma-Aldrich (USA). Chlorogenic acid, cryptochlorogenic acid, neochlorogenic acid, caffeic acid and 3-methyl-2-benzothiazolinone hydrazone (MBTH) hydrochloride monohydrate were all purchased from Macklin (China). N,N-dimethylformamide (DMF) was obtained from Sinopharm Chemical Reagent Co., Ltd (China). All the chemicals were of analytical grade.

\subsection{Methods}

1.2.1 MBTH Assay of the Reaction Products The reaction products were determined by MBTH assay described by Estelle Zeyer with minor modifications ${ }^{[32]}$. The reaction mixture consisting of $750 \mu \mathrm{L}$ of substrate solution, $750 \mu \mathrm{L}$ of $24 \mathrm{mmol} / \mathrm{L}$ aqueous MBTH solution, and $750 \mu \mathrm{L}$ of $100 \mathrm{mmol} / \mathrm{L}$ phosphate buffer (pH 7.2) containing $8 \%$ of DMF (analysis buffer) was pre-incubated at $37^{\circ} \mathrm{C}$ for $10 \mathrm{~min}$. Then, $750 \mu \mathrm{L}$ of 100 $\mathrm{U} / \mathrm{mL}$ enzyme solution was added to start the reaction. Absorbance was recorded over $20 \mathrm{~min}$ using a UV2100 spectrophotometer in $10-\mathrm{mm}$ quartz cuvettes. The maximum absorbance wavelength of the reactions for all the substrates was applied to all experiments below. Since the standards of the unstable reaction products were not commercially available, calibration curves were constructed after sufficient incubation of each substrate of specific concentration with excessive enzyme.

\subsubsection{Optimization of Reaction Conditions} reaction mixture consisting of $50 \mu \mathrm{L}$ of substrate solution, $50 \mu \mathrm{L}$ of $24 \mathrm{mmol} / \mathrm{L}$ MBTH and $50 \mu \mathrm{L}$ of analysis buffer was pre-incubated for $10 \mathrm{~min}$. The analysis buffer consisted of $100 \mathrm{mmol} / \mathrm{L}$ phosphate buffer containing $8 \%$ of DMF. The reaction was initiated by the addition of enzyme and terminated by adding $200 \mu \mathrm{L}$ ice-cold acetonitrile. After vortex and centrifugation, $200 \mu \mathrm{L}$ of the supernatant was applied for absorbance determination of the mixture system by microplate reader. Prior to kinetic study of tyrosinasecatalyzed oxidation of four polyphenols, the effect of incubation time, incubation buffer $\mathrm{pH}$, temperature and enzyme concentration was investigated to optimize the reaction conditions. The incubation time was optimized over a time range of 0 to $120 \mathrm{~min}$. The reaction was started by the addition of enzyme and the absorbance was immediately measured during the predefined time range by microplate reader. The effect of $\mathrm{pH}$ was studied using the four polyphenols as substrates in analysis buffer at a range of 4.5-7.5. The impact of temperature on enzymatic activity was optimized using a circulating water bath to control and maintain the temperature at $4,25,37,45,50,55$ 
and $60^{\circ} \mathrm{C}$, respectively. The mixture of substrate, MBTH and analysis buffer was pre-incubated for 10 min at appropriate temperature and then the reaction was initiated by adding the enzyme. For enzyme optimization, final concentrations of 5-500 U/mL, 10$1000 \mathrm{U} / \mathrm{mL}$ and $10-1500 \mathrm{U} / \mathrm{mL}$ tyrosinase were used for chlorogenic acid, neochlorogenic acid and other two substrates, respectively. The optimal incubation time, incubation buffer $\mathrm{pH}$, temperature and enzyme concentration obtained for tyrosinase-catalyzed oxidation were applied for the following kinetic study. 1.2.3 Kinetics of Tyrosinase-mediated Oxidation of Polyphenols The kinetics and substrates specificity of four polyphenols were examined under the final optimized incubation conditions. The reaction mixture consisting of $24 \mathrm{mmol} / \mathrm{L} \mathrm{MBTH}$, enzyme solution, $100 \mathrm{mmol} / \mathrm{L}$ phosphate buffer at a final volume of $150 \mu \mathrm{L}$ was pre-incubated for $10 \mathrm{~min}$. The reaction was initiated by the addition of $50 \mu \mathrm{L}$ substrate solution and terminated by the addition of $200 \mu \mathrm{L}$ ice-cold acetonitrile. The kinetics was determined in concentration ranges of 0.025 to $5 \mathrm{mmol} / \mathrm{L}$ and 0.1 to 10 $\mathrm{mmol} / \mathrm{L}$ for chlorogenic acid and other three substrates, respectively. GraphPad prism (version 7.0) was used for the non-linear regression analysis of the kinetic data to calculate the Michaelis-Menten constant $\left(\mathrm{K}_{\mathrm{m}}\right)$ and the apparent maximum rate $\left(\mathrm{V}_{\text {max }}\right)$. The catalytic constant $\left(k_{\text {cat }}\right)$ was calculated by dividing the apparent maximum rate by total amount of tyrosinase in units of inverse time.

1.2.4 Molecular Docking All molecular docking simulations were carried out using AutoDock Vina ${ }^{[33]}$. The tyrosinase structure was taken from our previous study ${ }^{[34]}$ which was constructed from crystal structure $2 \mathrm{Y} \mathrm{W}^{[35]}$ and optimized by quantum mechanics/ molecular mechanics $(\mathrm{QM} / \mathrm{MM})$ calculations. The four polyphenols, i.e., chlorogenic acid, neochlorogenic acid, cryptochlorogenic acid, and caffeic acid, were built up by using OpenBabel program ${ }^{[36]}$. Both the receptor and ligands were prepared by using AutoDock Tools $^{[37]}$. The search space was restricted to a box of $20 \times 20 \times 20 \AA$ (center $=5,25,103)$. This is sufficient to cover the binding pocket. Default values were used for all parameters. After docking, the top binding poses were selected for binding mode analysis.
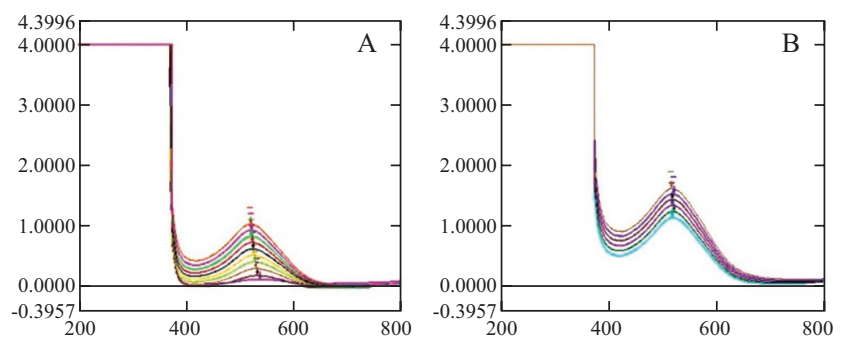

\section{RESULTS AND DISCUSSION}

\subsection{Analytical Assay Development for the Reaction Products}

The product resulting from the enzymatic oxidation of chlorogenic acid, the o-quinone, has been reported to be unstable substance ${ }^{[38]}$. In this situation, reliable analytical method becomes a prerequisite for such studies. Kanako et al detected the decrease in substrate concentration using a spectrophotometric method ${ }^{[39]}$. The analytical assay that focuses on the appearance of a stable chromatophoric compound demonstrates obvious advantages over methods that quantify substrate disappearance. Halis et al and Karla et al measured the o-quinones formation directly by the increased absorbance at maximum wavelength ${ }^{[31,40]}$. As is known, such unstable o-quinone suffers the attack of potent nucleophilic reagents and generates chromophoric adducts with clear stoichiometry. Spectrophotometric methods for measuring the activity of tyrosinase have been summarized and discussed in previously published review ${ }^{[41]}$. Among the commonly used nucleophilic reagents, MBTH and L-proline were used to characterize both monophenolase and diphenolase activity by measuring the accumulation of chromaphoric substance. In addition, Muñoz et al employed ascorbic acid as reducing agent to measure its disappearance as a result of oxidation by the quinones ${ }^{[38]}$.

As expected, we found the instability of o-quinones which quickly polymerized to colorful pigments. In this situation, the accurate enzyme activity results depended upon the reliability of analytical approaches. Compared with other analytical methods, the MBTH assay showed obvious advantages that MBTH conjugates of o-quinones were more stable than other conjugation forms. The MBTH assay provided a feasible way to detect the oxidation products by avoiding their losses caused by pigments production. In addition, the MBTH method was also proved to be more convenient and economical.

In the current study, we established a sensitive and effective method using $\mathrm{MBTH}^{[32]}$ to trap the o-quinone formed in the tyrosinase-catalyzed oxidation. As shown in fig. 1, the maximum absorbance wavelength of the reaction products of chlorogenic acid,
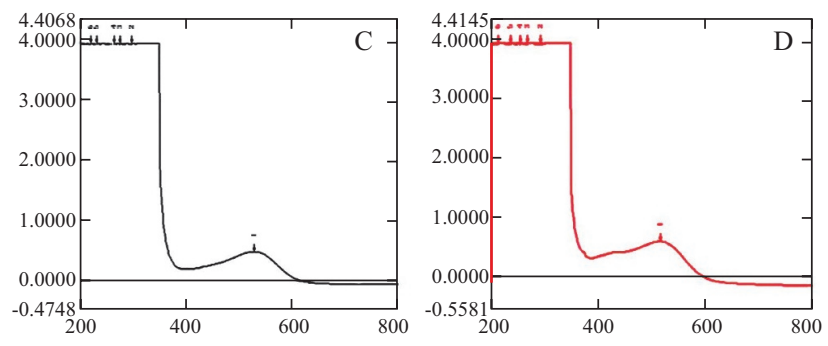

Fig. 1 The absorbance wavelength curve of four reaction products of polyphenols with tyrosinase (A: chlorogenic acid, B: cryptochlorogenic acid, C: neochlorogenic acid, D: caffeic acid) 
cryptochlorogenic acid, neochlorogenic acid and caffeic acid was 521, 526, 530, $516 \mathrm{~nm}$, respectively. The four polyphenols reacted with excessive enzymes under the final optimized conditions. The calibration curves were found to be linear in corresponding concentration ranges of $0-100 \mu \mathrm{mol} / \mathrm{L}$ and $0-250 \mu \mathrm{mol} / \mathrm{L}$ for neochlorogenic acid and other three substrates, respectively (fig. 2). The typical slope, intercept and correlation coefficient of calibration curves varied among substrates of interest (fig. 2). As expected, when the substrates concentration reached a certain higher level above the upper limit of quantification of the calibration curve, the absorbance
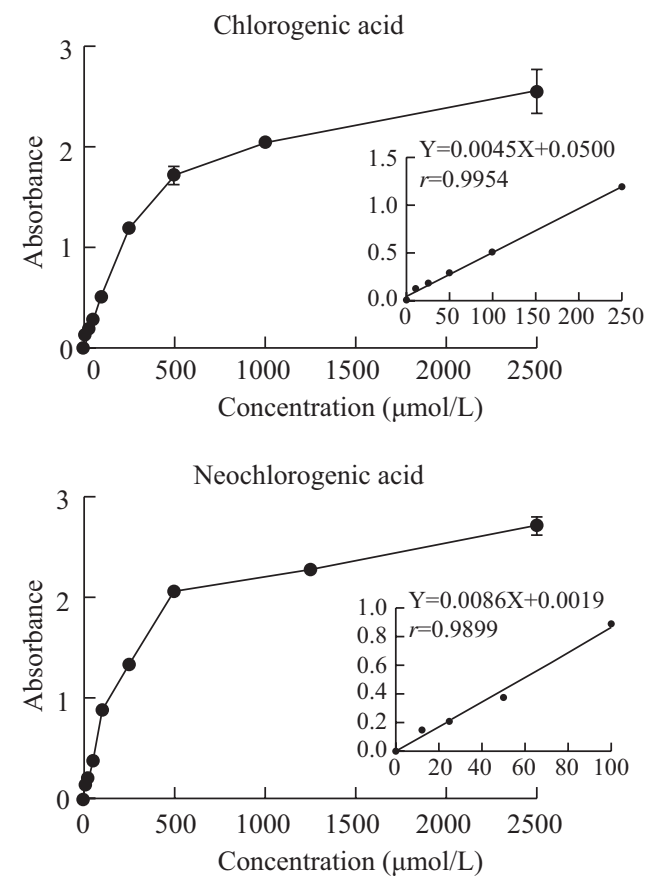

tends to be saturated.

\subsection{Condition Optimization for Tyrosinase Activity}

We investigated the effect of incubation time, incubation buffer $\mathrm{pH}$, temperature and enzyme concentration on tyrosinase-catalyzed oxidation of four polyphenols. To ensure that kinetic study was performed under linear conditions, the effect of incubation time on the product amount directly measured by the absorbance was studied over different time range for specific substrate. As shown in fig. 3 , the absorbance increased linearly with the incubation time of $10,15,30$ and 80 min for caffeic acid, chlorogenic
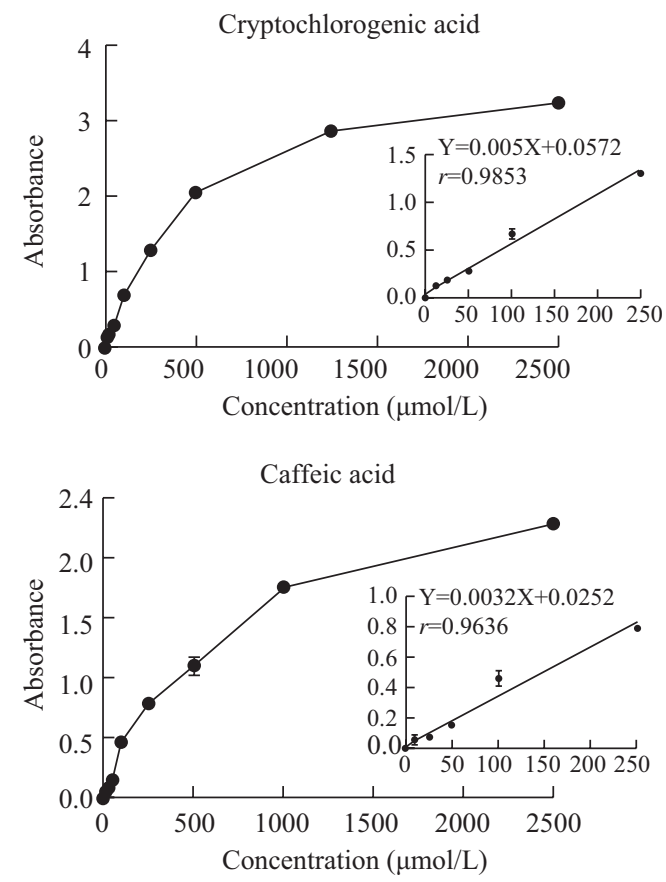

Fig. 2 The calibration curve of the four polyphenols reacted with excessive enzymes under the final optimized conditions
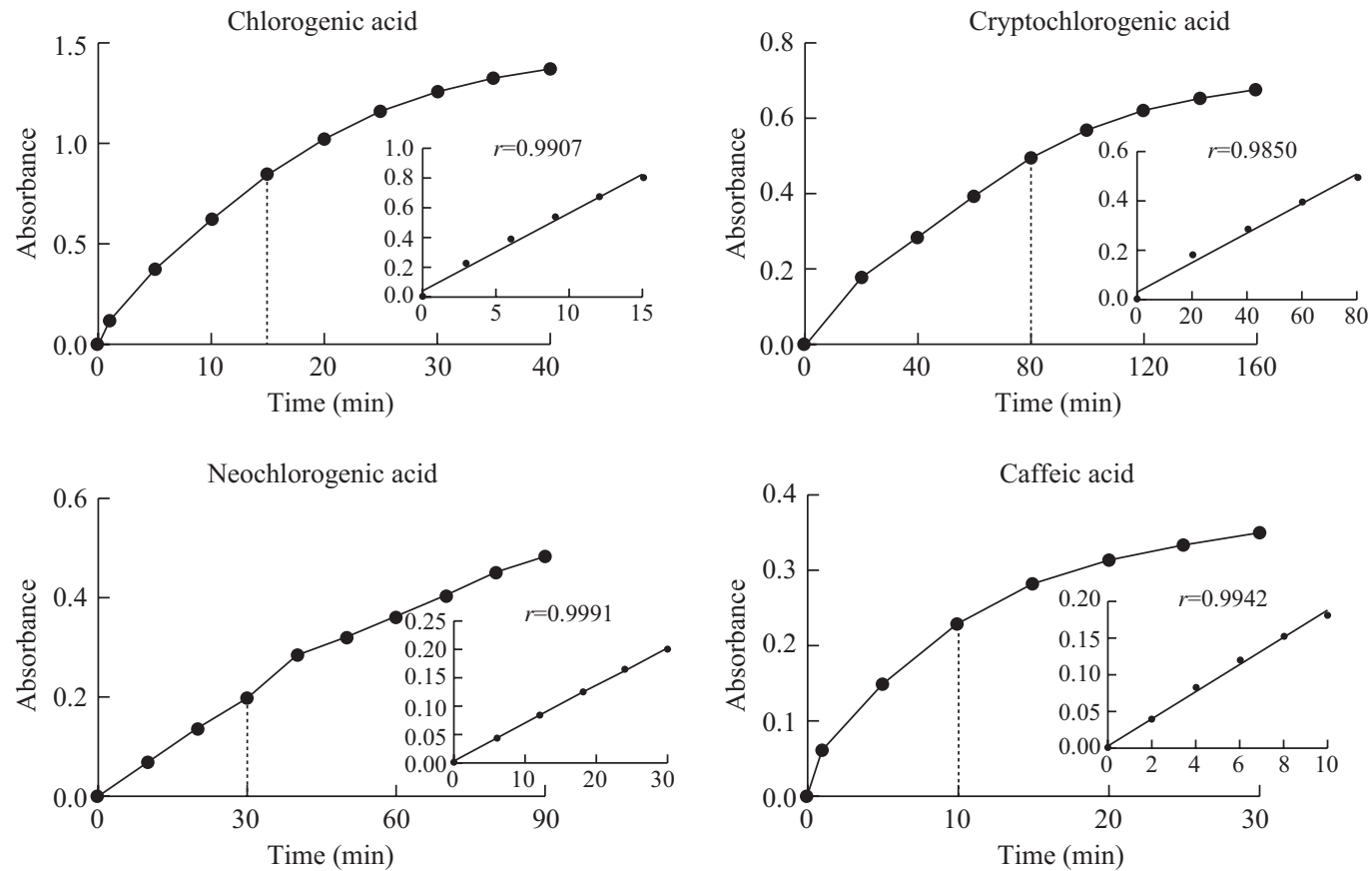

Fig. 3 Incubation time optimization for tyrosinase mediated oxidation of four polyphenols 
acid, neochlorogenic acid and cryptochlorogenic acid, respectively. 10-, 15-, 30- and 80-min incubation time was selected for the kinetic studies of the above mentioned compounds, because the oxidation rate was linear up to these time points and adequate amount of oxidation product was generated for spectrophotometric measurement. The difference of optimum time among the four polyphenol substrates may be attributed to the different structure of polyphenols, which may lead to different affinity with enzymes.

Polyphenol oxidase is a protein containing copper ions. Inappropriate $\mathrm{pH}$ can affect the activity of polyphenol oxidase through denaturation of copper ions and proteins. As shown in fig. 4, the $\mathrm{pH}$ of the incubation buffer seems to have an obvious influence on the reaction rate. For chlorogenic acid, enzyme activity was decreased in incubation buffer with increased $\mathrm{pH}$, while enzyme activity was increased when $\mathrm{pH}$ was greater than 6.5 for cryptochlorogenic acid. Unlike chlorogenic acid and cryptochlorogenic acid, peak enzyme activity was observed at $\mathrm{pH} 5.5$ and 6.0 for neochlorogenic acid and caffeic acid respectively. Different optimum $\mathrm{pHs}$ for polyphenol oxidase derived from various sources have been reported in previously published literature ${ }^{[31,42-45]}$. For example, optimum $\mathrm{pH}$ with chlorogenic acid substrate was found to be phosphate buffer $\mathrm{pH} 5.5$ for polyphenol oxidase obtained from dill (Anethum graveolens) ${ }^{[31]}$. The inconsistency between previous and our findings might be due to the source and the purity of the enzyme as well as difference in ionic strength of the incubation buffer ${ }^{[31,42]}$. Taken together, the optimum $\mathrm{pH}$ of 6.0 was chosen for chlorogenic acid, cryptochlorogenic acid and caffeic acid, while the optimum $\mathrm{pH}$ of 5.5 was selected for neochlorogenic acid.
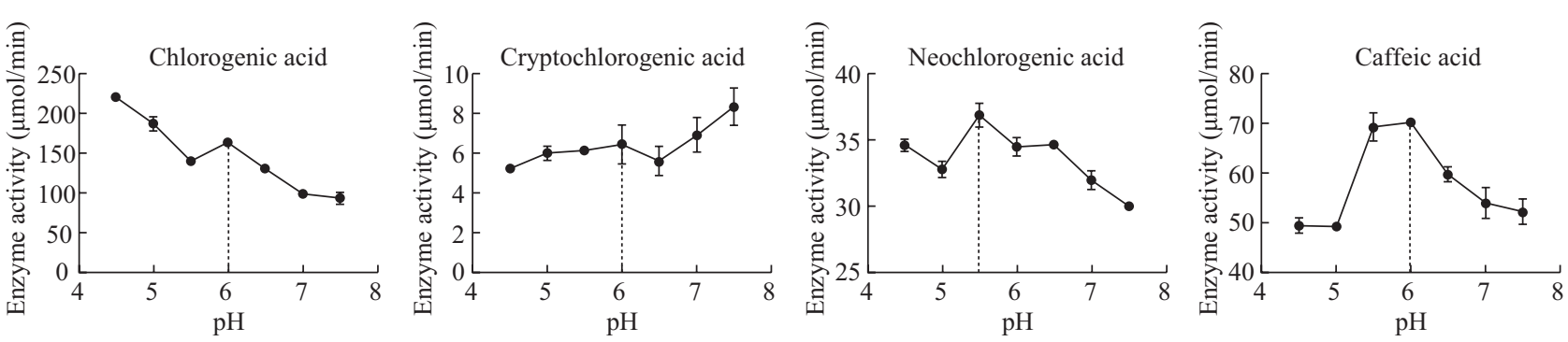

Fig. 4 The $\mathrm{pH}$ optimization for tyrosinase mediated oxidation of four polyphenols
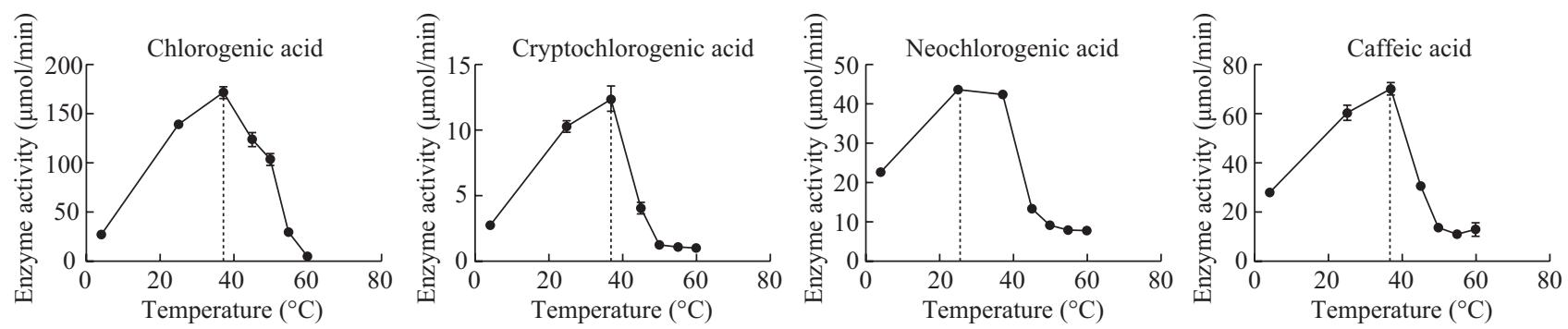

Fig. 5 The temperature optimization for tyrosinase mediated oxidation of four polyphenols

An increasing number of active enzyme molecules was associated with the increase of temperature. However, the denaturation of enzyme would be aggravated with the gradual increase of temperature, leading to the gradual loss of enzyme catalytic activity. Figure 5 shows that with the rise of temperature, the enzyme activity increased gradually when chlorogenic , cryptochlorogenic acid and caffeic acid were as substrates, and reached the maximum activity , which may be due to the fact that an increase experature to a certain extent causes the enzyme the a catalytic conformation in favor of the stresteresty, it was found that the optimum derived polyphenol oxidase was used. This might be also explained by the difference in the source and the purity of the enzyme as well as the ionic strength of incubation buffer ${ }^{[31,42]}$. The enzyme activity of neochlorogenic acid at $25^{\circ} \mathrm{C}$ is slightly higher than that at $37^{\circ} \mathrm{C}$. As the temperature continues to rise, however, the active conformation of the enzyme might be altered and the enzyme activity declined. When the temperature reached $60^{\circ} \mathrm{C}$, at least 5 -fold decrease in enzyme activity was observed for all four polyphenols of interest. Therefore, the optimum temperature of $25^{\circ} \mathrm{C}$ and $37^{\circ} \mathrm{C}$ was chosen for neochlorogenic acid and other three polyphenols of interest, respectively.

When the enzyme concentration was low, the enzyme activity increased linearly with the enzyme concentration since the substrate was overabundant at low enzyme concentrations (fig. 6). The reaction product generation rate was saturated once the enzyme concentration reached a certain high level (fig. 6). The explanation might be that under high enzyme concentration condition, substrate was not sufficient for 
enzyme molecule binding and thus substrate-limited saturation was observed. Good linear relationship was achieved between 0-100 U/mL (chlorogenic acid), 0-80 U/mL (cryptochlorogenic acid), 0-100 U/mL (neochlorogenic acid), and 0-100 U/mL (caffeic acid), respectively.

\subsection{Kinetic Analysis of Tyrosinase-catalyzed Oxida- tion of Polyphenols}

The kinetics of the four polyphenols was determined over pre-defined concentration range under the final optimal incubation conditions. The rate of product formation with respect to the substrates concentration demonstrated nonlinear Michaelis-Menten kinetics for four polyphenols (fig. 7). The Michaelis-Menten
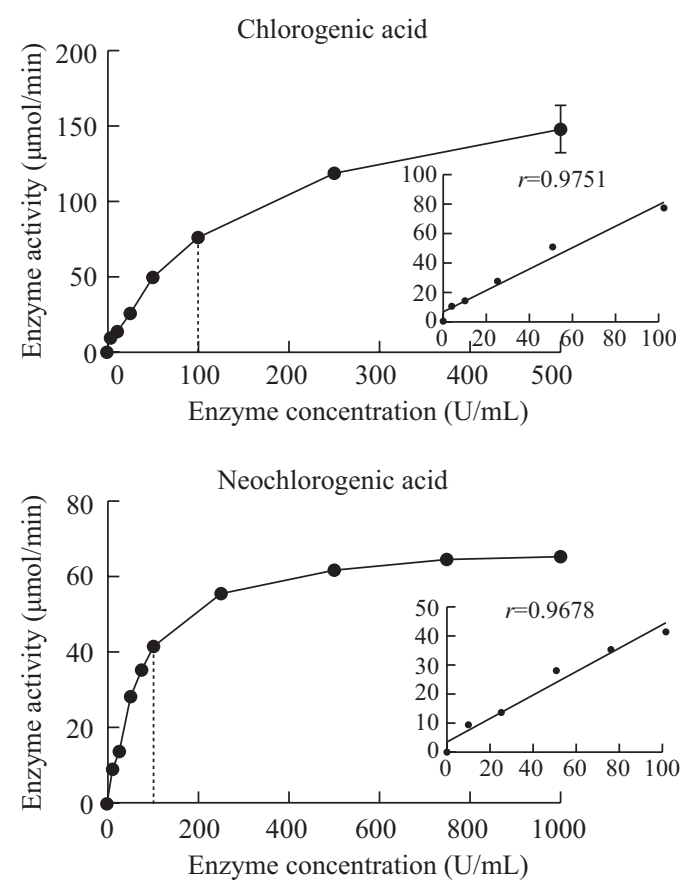

parameters including apparent $\mathrm{K}_{\mathrm{m}}$ and $\mathrm{V}_{\text {max }}$ as well as catalytic constant $k_{\text {cat }}$ and $k_{c a t} / \mathrm{K}_{\mathrm{m}}$ are listed in table 1 . As shown in table 1, mushroom tyrosinase has better affinity towards chlorogenic acid $\left(\mathrm{K}_{\mathrm{m}}=0.06 \mathrm{mmol} / \mathrm{L}\right)$ than other three substrates, as indicated by the high $\mathrm{V}_{\max } / \mathrm{K}_{\mathrm{m}}$ ratio. Our finding was in good agreement with previously reported $\mathrm{K}_{\mathrm{m}}$ of chlorogenic acid using dill-derived polyphenol oxidase ${ }^{[31]}$. When $\mathrm{V}_{\max }$ values are compared for the four substrates of interest, it was also found that $\mathrm{V}_{\max }$ for chlorogenic acid was higher than that for other three substrates. The parameter of catalytic efficiency $\left(k_{c a t}\right.$ and $\left.k_{c a t} / \mathrm{K}_{\mathrm{m}}\right)$ indicated that the tyrosinase-catalyzed oxidation of chlorogenic acid has the highest catalytic efficiency, which is 8- to 15 -fold
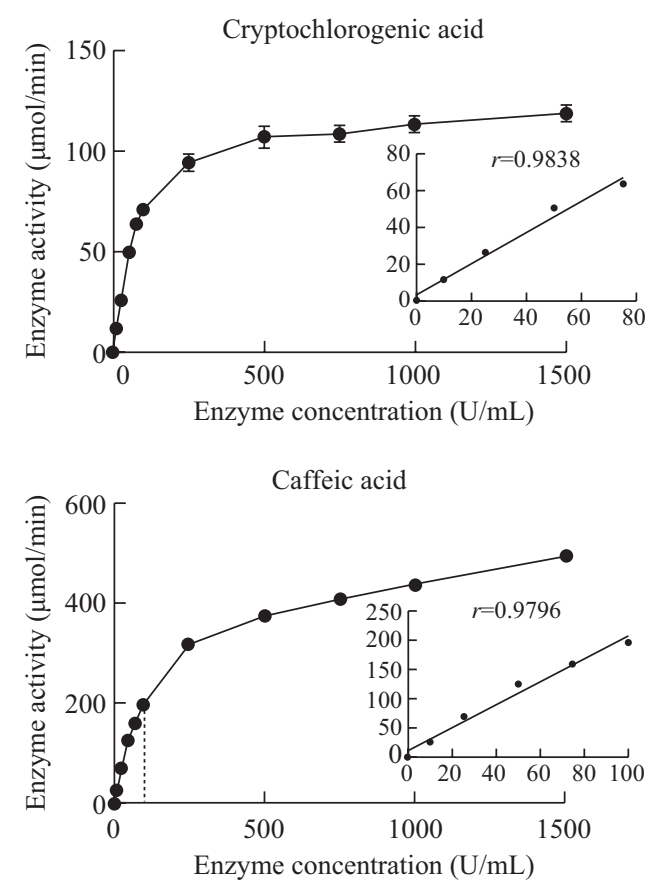

Fig. 6 Enzyme concentration optimization for tyrosinase mediated oxidation of four polyphenols.

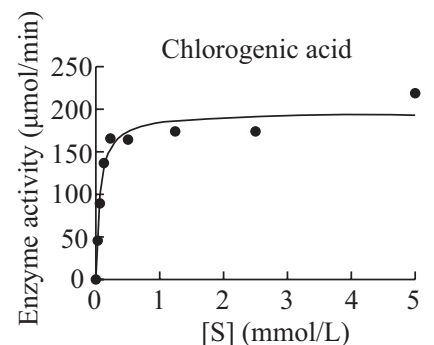

$[\mathrm{S}](\mathrm{mmol} / \mathrm{L})$

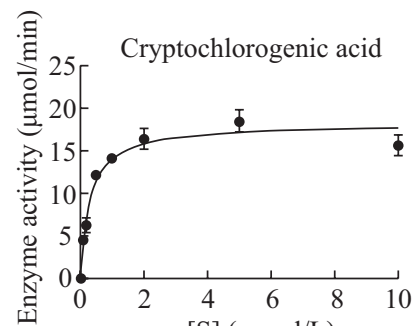

$[\mathrm{S}](\mathrm{mmol} / \mathrm{L})$

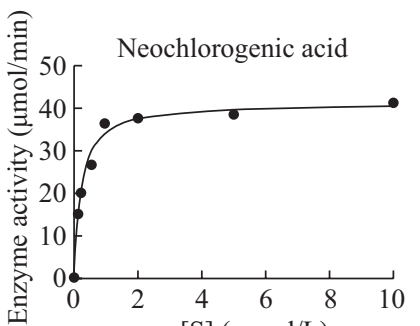

$[\mathrm{S}](\mathrm{mmol} / \mathrm{L})$

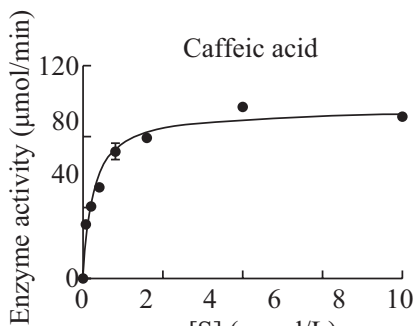

$[\mathrm{S}](\mathrm{mmol} / \mathrm{L})$

Fig. 7 Kinetics of tyrosinase-catalyzed oxidation of the four polyphenols

Data are presented as mean \pm standard deviation $(n=3)$.

Table 1 The kinetics of substrates oxidized by tyrosinase

\begin{tabular}{lcccc}
\hline & Chlorogenic acid & Cryptochlorogenic acid & Neochlorogenic acid & Caffeic acid \\
\hline $\mathrm{V}_{\max }(\mathrm{mmol} / \mathrm{L} / \mathrm{min})$ & 0.20 & 0.18 & 0.41 & 0.95 \\
$\mathrm{~K}_{\mathrm{m}}(\mathrm{mmol} / \mathrm{L})$ & 0.06 & 0.29 & 0.21 & 0.31 \\
$\mathrm{~V}_{\max } / \mathrm{K}_{\mathrm{m}}\left(\mathrm{min}^{-1}\right)$ & 3.27 & 0.06 & 0.20 & 0.31 \\
$k_{c a t}\left(\mathrm{~min}^{-1}\right)$ & 6335.48 & 589.35 & 1330.97 & 3059.03 \\
$k_{c a t} / \mathrm{K}_{\mathrm{m}}(\mathrm{L} / \mathrm{mmol} / \mathrm{min})$ & 105591.33 & 2032.24 & 6337.95 & 9867.83 \\
\hline
\end{tabular}


higher than that of the other 3 polyphenols. Except for the caffeic acid, other three compounds are isomers to each other. They are the esters of caffeic acid and quinic acid. The differences of these polyphenol isomers lie only in the esterification sites of quinine (see the first column in fig. 8). However, such structurally similar polyphenols exhibit significantly different kinetic behavior.

2.4 The Binding Modes of Polyphenols in Tyrosinase

It's of great interest to understand why tyrosinasecatalyzed oxidation is much more specific to chlorogenic acid than to the other polyphenols. Shown in fig. 8 are the chemical structures of polyphenols and binding modes obtained from molecular docking. The

(A) Chlorogenic acid

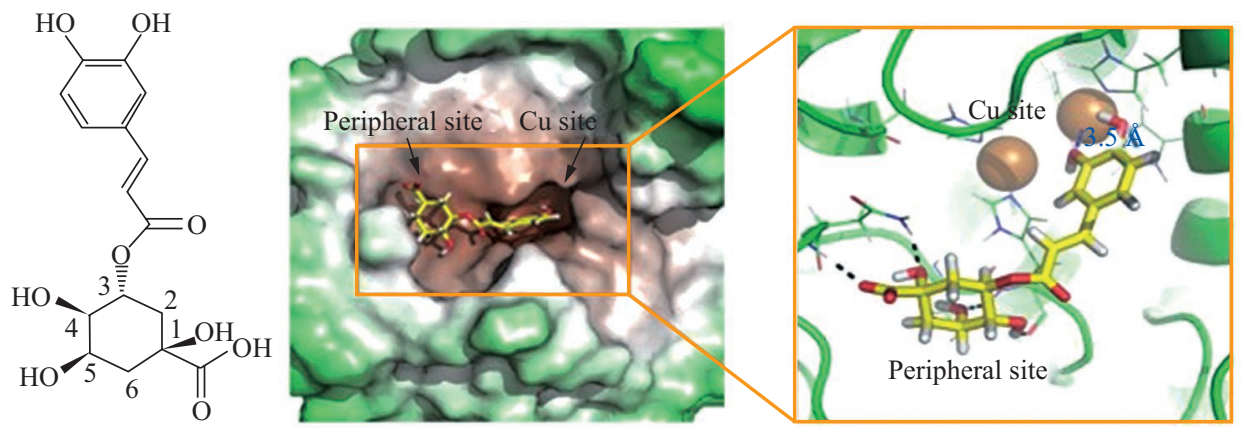

(B) Cryptochlorogenic acid<smiles>O=C(/C=C/c1ccc(O)c(O)c1)O[C@H]1[C@H](O)C[C@@H](O)[C@@](O)(C(=O)O)C[C@H]1O</smiles>

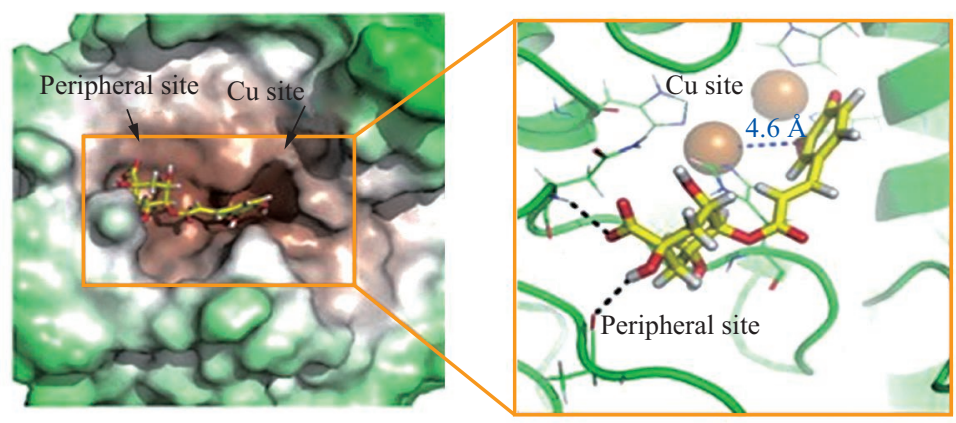

(C) Neochlorogenic acid
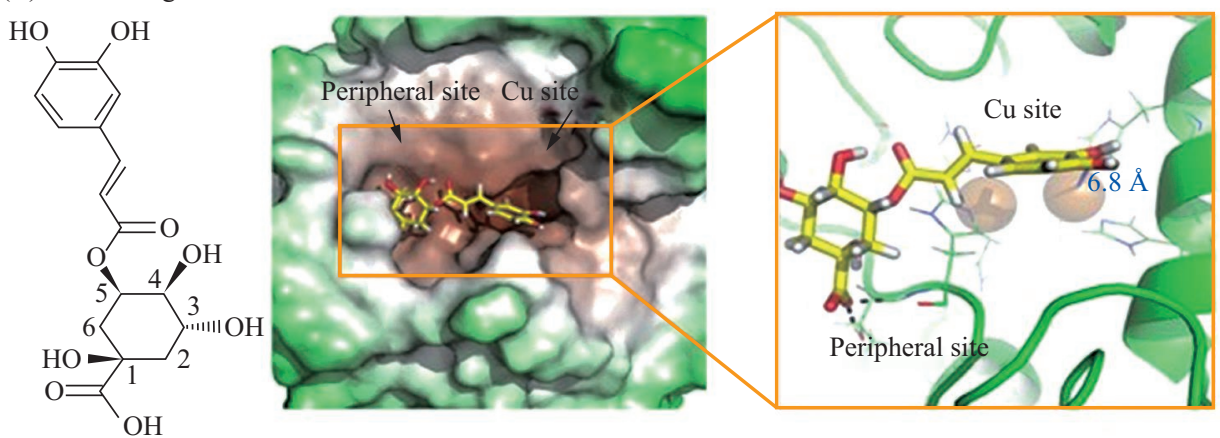

(D) Caffeic acid
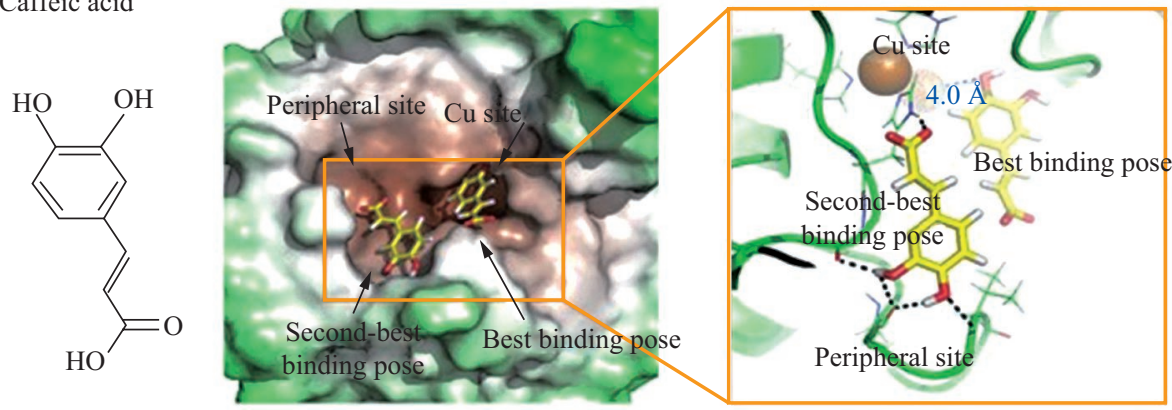

Fig. 8 The binding modes of (A) chlorogenic acid, (B) cryptochlorogenic acid, (C) neochlorogenic acid, and (D) caffeic acid in tyrosinase binding pocket obtained from molecular docking.

The carbon atoms of protein and ligands were colored with green and yellow, respectively. The copper, oxygen, nitrogen, and hydrogen atoms were colored with brown, red, blue, and white, respectively. The hydrogen bonds were rendered by black dash lines. The distances between phenol oxygen and the closest copper ion were labeled by blue dash lines. 
binding pocket consisted of two binding sites, which are labeled as $\mathrm{Cu}$ site and peripheral site, respectively. The $\mathrm{Cu}$ site contains the two copper ions, and the adjacent peripheral site is formed by residues $81-86$ and 321-324. As expected, all 4 polyphenols bind at the $\mathrm{Cu}$ site with their caffeic groups, in which the phenol groups point toward the copper ions. This observation is consistent with the fact that it is the phenol groups being oxidized. In addition to $\mathrm{Cu}$ site, chlorogenic acid and its isomers occupy the peripheral site with their quinic group (see the second column of fig. 8). The details of quinic group binding at the peripheral site (see the last column of fig. 8) show that chlorogenic acid forms 4 hydrogen bonds with the peripheral site, whereas neochlorogenic acid and cryptochlorogenic acid each forms only 2 hydrogen bonds. Clearly, chlorogenic acid binds tighter than the other two isomers, which explains why chlorogenic acid has the smallest $\mathrm{K}_{\mathrm{m}}$ value. As for caffeic acid, its molecular size is much smaller than other three substrates. It is thus not surprising to see that the affinity of caffeic acid is smaller than other three polyphenols, which results in the largest $\mathrm{K}_{\mathrm{m}}$ value among all 4 polyphenols. Also, the second-best binding pose of caffeic acid, which is shown in fig. $8 \mathrm{D}$, suggests a larger $\mathrm{K}_{\mathrm{m}}$ value. The docking score of the second-best binding pose is only $0.7 \mathrm{kcal} / \mathrm{mol}$ higher than that of the best binding mode. The ability to bind also the peripheral site indicates that part of caffeic acids is unable to participate the oxidation reaction, and thus increases the observed $\mathrm{K}_{\mathrm{m}}$ value.

The distance between the phenol oxygen and the closest copper ion $\left(\mathrm{d}_{\mathrm{O}-\mathrm{Cu}}\right)$ may reflect how fast the oxidation reaction proceeds. The closer the distance is, the more likely the oxidation proceeds faster. As seen from the last column of fig. 8 , the $\mathrm{d}_{\mathrm{O}-\mathrm{Cu}}$ for chlorogenic acid is $3.5 \AA$. It is significantly smaller than those for the other 3 polyphenols, suggesting that it is easier for chlorogenic acid to reach the transition state. Therefore, chlorogenic acid exhibits the largest $k_{c a t}$ value. Neochlorogenic acid and cryptochlorogenic acid have larger $\mathrm{d}_{\mathrm{O}-\mathrm{Cu}}$ and smaller $k_{\text {cat }}$. This is most likely due to the different binding modes of quinic groups at the peripheral site, which hinders the caffeic groups of neochlorogenic acid and cryptochlorogenic acid to more closely approach the copper ions. Interestingly, the best binding pose of caffeic acid displays a $\mathrm{d}_{\mathrm{O}-\mathrm{Cu}}$ of $4.0 \AA$ that is shorter than that of neochlorogenic acid and cryptochlorogenic acid. However, oxidation of caffeic acid is still the slowest one, suggesting that the substituent effect is very important in the tyrosinasecatalyzed oxidation reaction.

In summary, more specificity of tyrosinase to chlorogenic acid is majorly because of the tighter binding of chlorogenic acid at the peripheral site, which concurrently enables it to more closely approach the copper ions. In light of this, the rational design of highaffinity inhibitors against tyrosinase may focus on the binding of both the $\mathrm{Cu}$ site and peripheral site. Natural bioactive components such as phenolic compounds might be good substrates or inhibitors of tyrosinase due to possible interaction between phenolic compounds and the copper ions of tyrosinase. Because tyrosinase plays an important role in melanin synthesis, phenolic compounds such as chlorogenic acid might be widely used in health care and cosmetic products.

\section{CONCLUSION}

In this study, we established an MBTH assay to trap the o-quinone formed in the tyrosinase-catalyzed oxidation for enzyme kinetic study of the four polyphenols, i.e., chlorogenic acid, cryptochlorogenic acid, neochlorogenic acid, and caffeic acid. This analytical method overcomes the problem that the unstable o-quinone occurring from the enzyme oxidation of polyphenols negatively affected the quantification of the reaction product. The effect of experimental factors such as incubation time, $\mathrm{pH}$, temperature and enzyme concentration were investigated and optimal incubation conditions would be applied for kinetics characterization of the four polyphenols. The optimum incubation time, buffer $\mathrm{pH}$, temperature and enzyme level varied among substrates. Our kinetic data show that tyrosinase has the greatest affinity to chlorogenic acid compared with its isomers and caffeic acid. The catalytic efficiency with chlorogenic acid is 8- to 15fold higher than that with the other 3 polyphenols. The reason for such a great substrate specificity has been elucidated by our molecular docking results. The tight binding of chlorogenic acid at the peripheral site, which concurrently enables it to more closely approach the copper ions, should be the major reason for the specificity to chlorogenic acid. In light of this, the rational design of high-affinity inhibitors against tyrosinase may focus on the binding of both the $\mathrm{Cu}$ site and peripheral site.

\section{Open Access}

This article is licensed under a Creative Commons Attribution 4.0 International License https://creativecommons.org/licenses/by/4.0/), which permits use, sharing, adaptation, distribution and reproduction in any medium or format, as long as you give appropriate credit to the original author(s) and the source, provide a link to the Creative Commons licence, and indicate if changes were made. The images or other third party material in this article are included in the article's Creative Commons licence, unless indicated otherwise in a credit line to the material. If material is not included in the article's Creative Commons licence and your intended use is not permitted by statutory regulation or exceeds the permitted use, you will need to obtain permission directly from the copyright holder. To view a copy of this 
licence, visit http://creativecommons.org/licenses/by/4.0/.

\section{Conflict of Interest Statement}

The authors declare no conflict of interest.

\section{REFERENCES}

1 Działo M, Mierziak J, Korzun U, et al. The Potential of Plant Phenolics in Prevention and Therapy of Skin Disorders. Int J Mol Sci, 2016,17(2):160-200

2 Randhir R, Lin YT, Shetty K. Phenolics, their antioxidant and antimicrobial activity in dark germinated fenugreek sprouts in response to peptide and phytochemical elicitors. Asia Pac J Clin Nutr, 2004,13(3):295-307

3 Kolayli S, Kucuk M, Duran C, et al. Chemical and Antioxidant Properties of Laurocerasus officinalis Roem. (Cherry Laurel) Fruit Grown in the Black Sea Region. J Agric Food Chem, 2003,51(25):7489-7494

4 Can Z, Dincer B, Sahin H, et al. Polyphenol oxidase activity and antioxidant properties of Yomra apple (Malus communis L.) from Turkey. J Enzyme Inhib Med Chem, 2014,29(6):829-835

5 Nabavi SF, Tejada S, Setzer WN, et al. Chlorogenic Acid and Mental Diseases: From Chemistry to Medicine. Curr Neuropharmacol, 2017,15(4):471-479

6 Olthof MR, Hollman PCH, Katan MB. Chlorogenic acid and caffeic acid are absorbed in humans. J Nutr, 2001,131(1):66-71

7 Tajik N, Tajik M, Mack I, et al. The potential effects of chlorogenic acid, the main phenolic components in coffee, on health: a comprehensive review of the literature. Eur J Nutr, 2017,56(7):2215-2244

8 Heitman E, Ingram DK. Cognitive and neuroprotective effects of chlorogenic acid. Nutr Neurosci, 2017,20(1): 32-39

9 Zhou Y, Ruan Z, Wen YM, et al. Chlorogenic acid from honeysuckle improves hepatic lipid dysregulation and modulates hepatic fatty acid composition in rats with chronic endotoxin infusion. J Clin Biochem Nutr, 2016,58(2):146-155

10 Liang HQ, Yang JE, Tang JM, et al. Optimization of dosage ratio of chlorogenic acid and gardenia glycosides in the treatment of rats with fatty liver disease induced by high-fat feed. J Tradit Chin Med, 2016,36(5):683688

11 John RS, Leah W, Devin JM, et al. Analysis of the Polyphenols of Tobacco Using Pressurized Liquid Extraction (PLE) and Ultra Performance Liquid Chromatography With Electrospray Ionization Tandem Mass Spectometric Detection (UPLC-ESI-MS/ MS). Beitr Tabakforsch Int, 2017,27(8):195-207

12 Xi Y, Fan XG, Zhao HD, et al. Postharvest fruit quality and antioxidants of nectarine fruit as influenced by chlorogenic acid. Food Sci Technol, 2017,75(10):537544

13 Huang S, Wang LL, Xue NN, et al. Chlorogenic acid effectively treats cancers through induction of cancer cell differentiation. Theranostics, 2019,9(23):67456763

14 Amato A, Caldara G-F, Nuzzo D, et al. NAFLD and Atherosclerosis Are Prevented by a Natural Dietary Supplement Containing Curcumin, Silymarin, Guggul,
Chlorogenic Acid and Inulin in Mice Fed a High-Fat Diet. Nutrients, 2017,9(5):492-504

15 Ding Y, Cao Z, Cao L, et al. Antiviral activity of chlorogenic acid against influenza A (H1N1/H3N2) virus and its inhibition of neuraminidase. Sci Rep, 2017, 7(1):45723-45733

16 Cho AS, Jeon SM, Kim MJ, et al. Chlorogenic acid exhibits anti-obesity property and improves lipid metabolism in high-fat diet-induced-obese mice. Food Chem Toxicol, 2010,48(3):937-943

17 Wang YJ, Wen J, Zheng WH, et al. Simultaneous determination of neochlorogenic acid, chlorogenic acid, cryptochlorogenic acid and geniposide in rat plasma by UPLC-MS/MS and its application to a pharmacokinetic study after administration of Reduning injection. Biomed Chromatogr, 2015,29(1):68-74

18 Magnani C, Isaac VLB, Correa MA, et al. Caffeic acid: a review of its potential use in medications and cosmetics. Anal methods, 2014,6(10):3203-3210

19 Gheibi N, Taherkhani N, Ahmadi A, et al. Characterization of inhibitory effects of the potential therapeutic inhibitors, benzoic acid and pyridine derivatives, on the monophenolase and diphenolase activities of tyrosinase. Iran J Basic Med Sci, 2015,18(2):122-129

20 García Jiménez A, García-Molina F, Teruel J, et al. Catalysis and inhibition of tyrosinase in the presence of cinnamic acid and some of its derivatives. Int $\mathrm{J}$ Biol Macromol, 2018,119(11):548-554

21 Chen QX, Kubo I. Kinetics of mushroom tyrosinase inhibition by quercetin. JAgric Food Chem, 2002,50(14): 4108-4112

22 Kubo I, Kinst-Hori I. Flavonols from saffron flower: Tyrosinase inhibitory activity and inhibition mechanism. J Agric Food Chem, 1999,47(10):4121-4125

23 Kubo I, Kinst-Hori I, Chaudhuri SK, et al. Flavonols from Heterotheca inuloides: Tyrosinase inhibitory activity and structural criteria. Bioorg Med Chem, 2000,8(7):1749-1755

24 Kubo I, Yokokawa Y, Kinsthori I. Tyrosinase Inhibitors from Bolivian Medicinal Plants. J Nat Prod, 1995,58(5): 739-743

25 Wang Y, Zhou YF, Shen N, et al. Identification of a Homozygous Missense Mutation in the TYR Gene in a Chinese Family with OCA1. Curr Med Sci, 2018,38(5): 932-936

26 Maeda Ki, Fukuda M. In vitro effectiveness of several whitening cosmetic components in human melanocytes. J Soc Cosmet Chem, 1991,42(6):361-368

27 Palumbo A, Dischia M, Misuraca G, et al. Mechanism of inhibition of melanogenesis by hydroquinone. Biochim Biophys Acta, 1991,1073(1):85-90

28 Baharav E, Merimski O, Shoenfeld Y, et al. Tyrosinase as an autoantigen in patients with vitiligo. Clin Exp Immunol, 1996,105(1):84-88

29 Jordan AM, Khan TH, Osborn HMI, et al. Melanocytedirected enzyme prodrug therapy (MDEPT): Development of a targeted treatment for malignant melanoma. Bioorg Med Chem, 1999,7(9):1775-1780

30 Jordana AM, Khan TH, Malkin H, et al. Melanocytedirected enzyme prodrug therapy (MDEPT): Development of second generation prodrugs for targeted treatment of malignant melanoma. Bioorg Med Chem, 
2001,9(6):1549-1558

31 Sakiroglu H, Ozturk AE, Pepe AE, et al. Some kinetic properties of polyphenol oxidase obtained from dill (Anethum graveolens). J Enzyme Inhib Med Chem, 2008,23(3):380-385

32 Zeyer E, Heuson E, Himber C, et al. Novel approach to identify phenoloxidases inhibitors: Optimization of spectrophotometric MBTH assay for high throughput use enzymatic assays and analysis. Food Control, 2018,93(11):83-91

33 Trott O, Olson AJ. Software News and Update AutoDock Vina: Improving the Speed and Accuracy of Docking with a New Scoring Function, Efficient Optimization, and Multithreading. J Comput Chem, 2010,31(2):455461

34 Zou CM, Huang W, Zhao GK, et al. Determination of the Bridging Ligand in the Active Site of Tyrosinase. Molecules, 2017,22(11):11-21

35 Ismaya WT, Rozeboom HJ, Weijn A, et al. Crystal Structure of Agaricus bisporus Mushroom Tyrosinase: Identity of the Tetramer Subunits and Interaction with Tropolone. Biochemistry, 2011,50(24):5477-5486

36 O'Boyle NM, Banck M, James CA, et al. Open Babel: An open chemical toolbox. J Cheminform, 2011,3(1):3346

37 Morris GM, Huey R, Lindstrom W, et al. AutoDock4 and AutoDockTools4: Automated Docking with Selective Receptor Flexibility. J Comput Chem, 2009, 30(16):2785-2791

38 Munoz J, Garcia-Molina F, Varon R, et al. Kinetic characterization of the oxidation of chlorogenic acid by polyphenol oxidase and peroxidase. Characteristics of the o-quinone. J Agric Food Chem, 2007,55(3):920-928

39 Amaki K, Saito E, Taniguchi K, et al. Role of Chlorogenic Acid Quinone and Interaction of Chlorogenic Acid Quinone and Catechins in the Enzymatic Browning of Apple. Biosci Biotechnol Biochem, 2011,75(5):829832

40 Batista KA, Batista GLA, Alves GL, et al. Extraction, partial purification and characterization of polyphenol oxidase from Solanum lycocarpum fruits. J Mol Catal B-Enzym, 2014,102(4):211-217

41 Garcia-Molina F, Munoz JL, Varon R, et al. A review on spectrophotometric methods for measuring the monophenolase and diphenolase activities of tyrosinase. J Agric Food Chem, 2007,55(24):9739-9749

42 Dogan M, Arslan O, Dogan S. Substrate specificity, heat inactivation and inhibition of polyphenol oxidase from different aubergine cultivars. Int J Food Sci Technol, 2002,37(4):415-423

43 Lee CY, Smith NL, Pennesi AP. Polyphenoloxidase from DeChaunac grapes. J Sci Food Agric, 1983,34(9):987991

44 Oktay M, Kufrevioglu I, Kocacaliskan I, et al. Polyphenoloxidase from Amasya Apple. J Food Sci, 1995,60(3):494-496

45 Wescheebeling P, Montgomery MW. Strawberry Polyphenoloxidase: Extraction and Partial Characterization. J Food Sci, 1990,55(5):1320-1324

(Received Mar. 30, 2020; revised Apr. 10, 2020) 